POLITIK IN DER REICHSKANZLEI 


\section{SCHRIFTENREIHE \\ DER VIERTELJAHRSHEFTE FÜR ZEITGESCHICHTE \\ NUMMER 3}

Im Auftrag des Instituts für Zeitgeschichte

herausgegeben von Hans Rothfels und Theodor Eschenburg

Redaktion: Martin Broszat 
HERMANN PÜNDER

\section{POLITIK IN DER REICHSKANZLEI}

AUFZEICHNUNGEN AUS DEN JAHREN 1929-1932

Herausgegeben von

THILO VOGELSANG

DEUTSCHE VERLAGS-ANSTALT

STUTTGART 
(ㄷ) 1961 Deutsche Verlags-Anstalt GmbH., Stuttgart. Gesetzt aus der Monotype Walbaum-Antiqua. Gesamtherstellung: Deutsche VerlagsAnstalt GmbH., Stuttgart. Printed in Germany 


\section{EINLEITUNG}

Auf den nachfolgenden Blättern kommt ein Mann zu Wort, der von 1926 bis 1932 als Staatssekretär der Reichskanzlei in Berlin wirkte und von 1948 bis 1949 als Oberdirektor und Vorsitzender des Verwaltungsrates des Vereinigten Wirtschaftsgebietes in Frankfurt a. M. entscheidende Vorarbeiten für die Neuformung der deutschen Staatlichkeit leistete. Er gehört damit zu jener Gruppe von Personen, in denen über dieÄraHitler hinaus ein Stück Kontinuität deutscher Politiksichtbar geworden ist.

Hermann Pünder entstammt einer angesehenen katholischen rheinischen Familie und wurde am 1. April 1888 in Trier geboren. Seine schon in jungen Jahren erkennbare juristische Begabung mag im Elternhause eine entscheidende Förderung durch den Vater erfahren haben, der vor dem Ersten Weltkriege als Reichsmilitärgerichtsrat dem Zweiten Senat des Reichsmilitärgerichts in Berlin angehörte. Mit 21 Jahren trat Pünder 1909 nach erfolgreichem Studium der Rechts- und Staatswissenschaften in Freiburg i. Br., London und Berlin als Referendar beim Amtsgericht in Angermünde ein; ein Jahr später erfolgte die Promotion. Der Ausbruch des Krieges, den er als Reserveoffizier des 2. Thüringischen Feldartillerie-Regiments Nr. 55 (Naumburg a. S.) bis zum Ende mitmachte, verzögerte die Zweite Staatsprüfung; im Frühjahr 1915 wurde er Gerichtsassessor. Dem Heimgekehrten eröffnete sich eine schnelle Karriere. Nach kurzen Beschäftigungen am Landgericht I Berlin und im preußischen Justizministerum wurde Pünder im September 1919 als Regierungsrat in das Reichsfinanzministerium berufen.

Bereits 1921 als Oberregierungsrat in der (,Haushalts“-)Abteilung I unter Ministerialdirektor von Schlieben tätig, stieg er 1923 zum Ministerialrat auf und erhielt im Oktober, als der bisherige Ernährungsminister Dr. Hans Luther im zweiten Kabinett Stresemann das Finanzressort übernahm, die Leitung des sog. „Spezialbüros des Ministers "1. In diese Zeit fiel die durch seine unmmittelbare Stellung zu Luther verstärkte Mitwirkung an der Stabilisierung der Währung. Diese Zusammenarbeit fand ihre Fortsetzung, als Luther Mitte Januar 1925 an die Spitze einer bürgerlichen Regierung von den Deutschen Demokraten bis zu den Deutschnationalen trat und Pünder, jetzt Ministerialdirektor, ihm in die Reichskanzlei nachfolgte. Das neue Arbeitsfeld ermöglichte ihm weite Einblicke in die allgemeinen innen- und außenpolitischen Fragen. Dadurch erlangte er jene Personenkenntnis und Sicherheit in der Behandlung politischer Situationen, die ohne Zweifel mit dazu beitrugen, daß er im Juli 1926 auf die erste Stelle innerhalb der Reichskanzlei, auf den Posten des Staatssekretärs, nachrückte.

Den Anlaß dazu hatte im Mai der plötzliche Rücktritt der Regierung Luther gegeben, die durch ein Minderheitskabinett „Luther ohne Luther" unter der Füh-

1 Handbuch für das Deutsche Reich 1924, S. 136. 
rung des zurückkehrenden Altreichskanzlers Dr. Wilhelm Marx (Zentrum) ersetzt wurde. Im Anschluß daran berührten die permanenten und stets nur von Fall zu Fall überbrückbaren Gegensätze zwischen Deutscher Volkspartei und Zentrum auch die Stellung des Staatssekretärs Dr. Kempner, der schon seit 1919 der Reichskanzlei angehörte und 1925 unter Luther in deren Leitung der Nachfolger Brachts geworden war. Kempner wich dem Druck der Parteien ${ }^{1}$ und der Auffassung, dem Kanzler Marx müsse ein Mitarbeiter zur Seite gestellt werden, der über gute Beziehungen zum Zentrum verfüge. $\mathrm{Da}$ man jedoch keinen geeigneten Politiker fand, entschied sich Marx - selber alter Beamter - schließlich für den jungen Verwaltungsfachmann Pünder, eine Wahl, zu der auch - nachdem nun einmal Kempner ausgeschieden war-Stresemann geraten hatte.

Die Reichskanzlei, am 18. Mai 1878 auf ausdrücklichen Wunsch Bismarcks durch Allerhöchste Kabinettsorder Wilhelms I. als die oberste Reichsbehörde und unmittelbares Organ des Reichskanzlers errichtet ${ }^{2}$, hatte seitdem nicht nur ständig an Umfang und politischer Bedeutung gewonnen ${ }^{3}$, sondern auch durch die Reichsverfassung von 1919 eine Erweiterung ihrer Aufgaben erfahren. So blieb sie zwar das „Zentralbüro des Reichskanzlers“, wurde aber darüber hinaus zu einer Behörde, welche zugleich die laufenden Geschäfte des neugeschaffenen Regierungskollegiums $\mathrm{zu}$ besorgen, also auf das Zusammenwirken der Ressorts in Übereinstimmung mit der Kabinettspolitik zu achten hatte. In dieser Schlüsselposition boten sich Möglichkeiten persönlicher politischer Initiative imEinvernehmen mitKanzler undMinistern.

Wenn sich Pünders Stellung als Staatssekretär, die ohnehin auf einem voraufgegangenen reichlichen Jahr Wilhelmstraßen-Erfahrung aufbauen konnte, alsbald in einem Maße festigte, daß er in der Weimarer Zeit dieses Amt am längsten, nämlich durch sechs Jahre bekleiden konnte, dann hing das mit zwei Dingen zusammen. Zum einen wirkte sich die Konsolidierung der Republik auch an der Spitze der Reichskanzlei aus. Mochten auch hinter der Bestallung des Mitgliedes der Zentrumspartei Dr. Pünder zum Chef dieser Behörde einige politische Wünsche stehen, so war dieser Vorgang dennoch nur mehr ein schwacher Ausläufer jener Bestrebungen in den vorhergehenden Jahren, die vom November 1918 bis 1926 der Reichskanzlei nacheinander acht Staatssekretäre beschert hatten. Entscheidend wurde vor allem, daß die zwar politische, aber von ihm selbst so genannte „rein sachliche" und überparteiliche Arbeit des Praktikers Pünder und sein daraus sich ergebendes Ansehen die nächsten Regierungswechsel von 1927, 1928 und $1930 \mathrm{zu}$ überdauern vermochten. Hinzu kam eine gewisse Abneigung des Reichskanzlers Marx, sich selber in internationalen Gesprächen und Tagungen, den Erscheinungs-

1 M. von Stockhausen, Sechs Jahre Reichskanzlei, Erinnerungen und Tagebuchnotizen 1922-1927, hrsg. von W. Görlitz, Bonn 1954, S. 224 und 226.

2 Zur Sache vgl. Zur Geschichte des Reichskanzlerpalais und der Reichskanzlei, hrsg. vom Staatssekretär der Reichskanzlei, Berlin 1928, S. 33 ff. Ferner: R. Morsey, Die oberste Reichsverwaltung unter Bismarck 1867-1890, Münster i. W. 1957, S. $219 \mathrm{ff}$.

3 Die Chefs der Reichskanzlei sollten ursprünglich die Stellung eines "Vortragenden Rats" bekleiden. Seit 1907 standen sie im Range eines Unterstaatssekretärs; 1920 wurde die Stelle eines „Staatssekretärs der Reichskanzlei" geschaffen. 
formen des neuen außenpolitischen Stils der $20 \mathrm{er}$ Jahre, zu engagieren (im Gegensatz etwa zu Luther, der an der Seite Stresemanns eine betonte persönliche Beteiligung an den Verhandlungen von Locarno gezeigt hat). So fügte es sich, daßPünder im September 1926 als Beobachter des Reichskanzlers und als anerkannter Mitarbeiter Stresemanns der deutschen Delegation angehörte, die den vom Außenminister mühselig erarbeiteten Eintritt des Reiches in den Völkerbund vollzog. Gleichfalls im folgenden Jahre (1927) war er Mitglied der für die Vollversammlung des Völkerbundes bestimmten Delegation. Was Stresemann damals in Genf an Verhandlungen mit Briand und Austen Chamberlain führte, fand (zur Orientierung des Kanzlers) seinen ersten schriftlichen Niederschlag in den Telegrammen und Aufzeichnungen, die Pünder laufend nach Berlin sandte ${ }^{1}$. Es ist das eine seiner speziellen Obliegenheiten geworden; er hat sie ebenso bei anderen Gelegenheiten, wie z. B. auf den beiden Haager Konferenzen 1929 und 1930, mit besonderer Aufmerksamkeit wahrgenommen $^{2}$. Auch an der Sommertagung des Völkerbundsrates 1929 in Madrid nahm Pünder zusammen mit Staatssekretär von Schubert als Mitarbeiter des von ihm verehrten Außenministers teil.

Als Stresemann am 3. Oktober 1929 starb, schien nicht nur das sogenannte „beste Jahrfünft" des Weimarer Staates (A. Schwarz) zu Ende gegangen zu sein. Auch die Tätigkeit Pünders an der Spitze der Reichskanzlei trat gewissermaßen in ihre zweite Halbzeit, in die Phase der Krisen erst des Parlamentes und dann der Republik selbst. Vielleicht hat Pünder die Bedeutung dieser Zäsur schon damals stark empfunden; jedenfalls setzt in diesen tragischen Oktobertagen das vorliegende Tagebuch ein.

Das handschriftliche Original der Aufzeichnungen überdauerte den Zweiten Weltkrieg und das harte Lebensschicksal Pünders zwischen 1933 und 1945. Das Tagebuch wurde 1948/49, nach Mitteilung des Autors ohne inhaltliche Änderung, in ein maschinenschriftliches Manuskript übertragen. Diese Ausfertigung hat Pünder im Frühjahr 1960 dem Institut für Zeitgeschichte für die Zwecke einer Veröffentlichung im Rahmen der "Schriftenreihe der Vierteljahrshefte für Zeitgeschichte" überlassen. Sie wurde von ihm mit geringfügigen handschriftlichen Korrekturen versehen, die ich in die Edition übernommen habe ${ }^{3}$. Dabei handelt es sich lediglich um Berichtigungen von Fehlern oder um erklärende, dem besseren Verständnis dienende Zusätze, nicht jedoch um Veränderungen, welche die originale Quellenaussage beeinträchtigen würden. Von der Veröffentlichung wurden rein private, mit den politischen Ereignissen in keinem Zusammenhang stehende Passagen aus-

1. Schreiben Stresemanns an Reichskanzler Marx vom 21. September 1927, in: G. Stresemann, Vermächtnis, hrsg. von H. Bernhard, Bd 3, Berlin 1933, S. 196.

2 Vgl. unten S. 38 f.

3 Ein Durchschlag dieser maschinenschriftlichen Ausfertigung - ohne die erwähnten Korrekturen und ohne Ergänzung schwer lesbarer Stellen des Originals - gelangte vor einigen Jahren zusammen mit anderen, auf den nachfolgenden Seiten des öfteren in den Fußnoten angezogenen Papieren in das Bundesarchiv, wo ex in Band 4 der Sammlung Dr. Hermann Pünder unter dem Titel "Tagebuchaufzeichnungen aus der Zeit vom Tode Stresemanns bis zum Beginn des Dritten Reiches 1929-1934" aufbewahrt wird. 
genommen. Bei der Kommentierung des Tagebuch-Textes ist mir sowohl von seiten des Autors als auch durch das Bundesarchiv Koblenz, insbesondere von Herrn Archivrat Dr. Vogel, eine umfassende Unterstützung zuteil geworden, für die ich an dieser Stelle meinen aufrichtigen Dank aussprechen möchte.

Als eine der wenigen ,nichtamtlichen" Quellen für die Geschichte der Großen Koalition und der Ära Brüning vermag das Tagebuch Pünders, selbst bei Berücksichtigung der zuweilen etwas unterschiedlichen Intensität im Rhythmus der Eintragungen, eine wesentliche Aussage zu bieten, zumal sich in sehr vielen Fällen mühelos ergänzende Verbindungen zu den übrigen Pünder-Papieren sowie den einschlägigen Akten aus dem Bestande Reichskanzlei herstellen lassen. Dem Betrachter werden aufschlußreiche Einblicke in den Alltag der obersten Zentralbehörde des Reiches geboten, und zwar für eineZeit, da in ihr wirklich Politik gemacht wurde : so etwa am 6. März 1931, als just an einem scheinbaren Höhepunkte der neuen, auf eine Zollunion mit Österreich eingestellten Bemühungen des Außenministers Curtius nun gleichzeitig die Reparationspolitik Brünings inauguriert wurde, welche, vorangetrieben durch den Zwang der Verhältnisse, sich schnell vom Spezialgebiet der Wirtschafts- und Finanzexperten zum Hauptthema der internationalen Beziehungen wandelte und alsbald gar den Kanzler selbst zu einem Außenpolitiker machte. In diesen Wochen und Monaten wurde die Reichskanzlei, wie z. B. der Ablauf der Bankenkrise im Hochsommer 1931 zeigt, zu einer Stätte der Begegnungen und Beratungen, in denen in unaufhörlichem Wechsel Probleme und politische Belastungen oftmals durch den Staatssekretär, fast immer mit seiner Mithilfe gemeistert wurden. Pünder, der sicher nicht unbedingt „ruhende“, aber von den Mitakteuren der Zeit wegen seiner Sachlichkeit geschätzte „Pol der Vernunft" (so der Ausspruch Ludwig Kaas'), arbeitete mit vorausschauender Umsicht, mit Geduld und Überzeugungskraft. Im Dezember 1929, als die von Schacht hochgespielte Ultimokrise bereits die Koalition zu gefährden drohte, riet Pünder zum „Frieden“ mit dem Reichsbankpräsidenten und setzte sich damit durch.

$\mathrm{Da} ß$ die Sozialdemokratie nach den Herbstwahlen 1930 für eine Tolerierungspolitik gegenüber dem Kabinett Brüning gewonnen werden konnte, ist zum großen Teil Pünders Verdienst. Und als der Staatssekretär sehen mußte, daß im Mai 1932 der Wandel in den Auffassungen Hindenburgs nicht mehr rückgängig zu machen war, versuchte er noch in letzter Minute eine Einschaltung Goerdelers als preuBischen Ministerpräsidenten oder gar als Kanzler zu erreichen, um Brüning wenigstens im Auswärtigen Amt zu halten und um den „Rechtsruck“, so gut es ging, zu entschärfen. Daß hinter der Berichterstattung des Tagebuches eine politische, selbstbewußte und temperamentvolle Persönlichkeit steht, die juristisches Können 'mit einem sehr lebhaften Gefühl für die Verantwortungsfülle des Amtes verband, wird der Leser bald bemerken. Pünders ausgeprägter Sinn für Personenbeziehungen haben den "jugendlichen " Staatssekretär, der mit 38 Lebensjahren an die Spitze der Reichskanzlei getreten war, vor allem auch befähigt, die Tätigkeit des Harmonisierens und des Ausgleichens zwischen Politikern und Ministern, zwischen Parteien und Institutionen auszuüben. 
Pünders sachliche Unbefangenheit gegenüber dem Instrument des Artikels 48 der Reichsverfassung rührt vermutlich bereits aus seiner früheren Zusammenarbeit mit Luther her; in einem mehr als dreißig Jahre später gehaltenen Vortrag ist in unseren Tagen diese Einstellung unter Berufung auf das Ermächtigungsgesetz anläßlich der Einführung der Rentenmark im November 1923 noch einmal ganz deutlich geworden ${ }^{1}$. Als Staatssekretär und erster Mitarbeiter des Reichskanzlers Müller hat Pünder gewiß nicht an der hintergründigen Vorbereitung der nachfolgenden Regierung teilgenommen, doch konnte er ohne weiteres die wesentlich kritischere Haltung eines Heinrich Brüning gegenüber „diesem " Parlament auch zu seiner eigenen machen. Sowohl vor wie nach dem 30. März 1930 verschloß er, um die in Verbindung mit dem Youngplan eingeleitete Wirtschafts- und Finanzgesetzgebung in Gang zu bringen, vor der Notwendigkeit einer Anwendung des Artikels 48 keinesfalls die Augen. Wie Brüning, Groener und Schleicher mag auch Pünder damals große Hoffnungen auf die - man muß schon sagen - Neukonstruktion einer gemäßigten arbeitsfähigen Rechten gesetzt haben.

$\mathrm{Da}$ der Reichstag der IV. Wahlperiode 1928 tatsächlich in keinem hohen Ansehen mehr stand, beweist die rote Mappe mit der Auflösungsorder Hindenburgs, die bereits am 3. April 1930, dem ersten Tage der Begegnung der neuen Regierung mit dem Reichstag, griffbereit auf der Ministerbank lag! So verbindet sich schließlich die Person Pünders mit den politischen Stileigentümlichkeiten der Brüningzeit, wie sie sich in mannigfacher Weise gezeigt haben : etwa im Wunsche nach einem Ermächtigungsgesetz im Sommer 1930, in dem schon Anfang Dezember 1930 erwogenen Plan, auf Brüning und Groener durch die Personalunion von Kanzler- und Auswärtigem Amt bzw. von Wehr- und Innenministerium die eigentliche politische Macht der Republik zu konzentrieren, und vor allem in dem Bestreben, auf dem Gebiete der Gesetzgebung und Verordnungen die überwiegende Initiative an die "Reichsführung " mitsamt „Fach"ministern und spezialisierter Bürokratie zu ziehen und andererseits den Reichsrat stärker an der Verantwortung zu beteiligen. Wenn indessen dennoch bei aller Distanzierung von der kaum mehr funktionierenden, stattdessen zunehmend blockierten Mehrheitsmechanik des Parlamentes die Beziehungen der Regierung zu den führenden Parteipolitikern unbelastet und die Parteien selbst als Repräsentanten des politischen Volkswillens im Grundsatz anerkannt blieben, dann ist solches ein Verdienst der beiden Brüning-Kabinette, an welchem Pünder seinen beachtlichen persönlichen Anteil gehabt hat.

Gemessen an Pünders Bereitschaft, unter Brüning die so oft besprochene Rechtsschwenkung der deutschen Politik mitzumachen, erscheint dem Leser seine Absage an Papen vom 2. Juni 1932 nur noch eindrucksvoller. Der Staatssekretär hatte eine gute Witterung für die kommenden Bedenkenlosigkeiten jener, die da zu erreichen wähnten, was dem "guten Heinrich“ ja doch nicht gelänge. Vor allem empörten ihn die Formen der kalten Isolierung Brünings und die hinter dem Rücken des Kanzlers auf Schleichers Initiative vorgenommene Vorbereitung neuer, angeb-

1 H. Pünder, Der Reichspräsident in der Weimarer Republik, Frankfurt a. M. und Bonn 1961, S. 25 f. 
lich sinnvollerer Konstellationen. War so für den in langen Jahren bewährten Mitarbeiter der Luther, Marx, Hermann Müller und Brüning an der Schalttafel der Reichspolitik keine Möglichkeit des Bleibens mehr gegeben, durfte er dennoch der Meinung sein, dem Staate Preußen in verantwortungsvoller Position unangefochten weiterdienen zu können. Die Übertragung des Regierungspräsidiums in Münster i. W. schien im Oktober 1932 diese Erwartung zu erfüllen. Aber schon acht Monate später wurde dem von ihm so hoffnungsvoll begrüßten Neuanfang wieder ein Ende gemacht. Ende Juni 1933 erfolgte - sinnigerweise aufGrund des Gesetzes zur „Wiederherstellung des Berufsbeamtentums" - die Entfernung aus dem Amt durch Spruch der neuen Herren Preußens, denn der Regierungspräsident, dem man sogar Aussichten auf das Oberpräsidium in Westfalen gemacht hatte, war bei seiner strikten Weigerung, der NSDAP beizutreten, geblieben.

Für Pünder begannen schwere Jahre. Seine weitere Familie mußte den verbrecherischen Machthabern des Dritten Reiches einen erheblichen Blutzoll leisten : am 30. Juni 1934. wurde derVetter Frau Pünders, Dr. Erich Klausener ${ }^{1}$, umgebracht; nach dem 20. Juli wurde ein Bruder seiner Frau, LeoStatz, durch Freisler wegen angeblicher „Heimtücke" zum Tode verurteilt und hingerichtet. Auch Pünder selbst hat wegen seiner Verbindungen zu Goerdeler am Ende des Krieges vor dem Volksgerichtshof gestanden. Zusammen mit anderen prominenten KZ-Häftlingen erlebte er im Mai 1945 am Pragser Wildsee in den Dolomiten die Befreiung.

Nach dem Kriege wurde die Kommunalpolitik Pünders neues Betätigungsfeld. Schon im Sommer 1945 einer der Begründer der westfälischen CDU in Münster i. W., war er im Spätherbst Oberbürgermeister von Köln geworden. Als erster Präsident des wiedererrichteten Deutschen Städtetages verhalf er diesem zu einem neuen Start und wirkte beratend an der Schaffung des Landes Nordrhein-Westfalen mit, indem er sich von Anfang an entschieden für dessen jetzige Grenze aussprach². Am 2. März 1948 wählte ihn der Wirtschaftsrat des Vereinigten Wirtschaftsgebietes („Bizone“) zum Oberdirektor und Vorsitzenden des Verwaltungsrates. Was Pünder hier zusammen mit anderen Politikern und Beamten in Frankfurt a. M. für die organisatorische Vorbereitung der zweiten deutschen Republik geleistet hat, muß einer späteren Betrachtung vorbehalten bleiben. Nach Konstituierung der Bundesrepublik wirkte er von 1949 bis 1957 im Bundestag als Abgeordneter der CDU und zweifacher AusschußVorsitzender weiter. Er gehörte schon im Herbst 1950 der ersten deutschen Delegation an, die an den Tagungen der Beratenden Versammlung des Europarates in Straßburg teilnahm. Im September 1952 ist Pünder zum 1. Vizepräsidenten der Gemeinsamen Versammlung der Europäischen Gemeinschaft für Kohle und Stahl gewählt worden. Dieses letzte Amt in einem an politischer Arbeit reichen Leben hat er bis 1958 ausgeübt.

Thilo Vogelsang

1 Vgl. unten Anm. 55.

2 H. Pünder, Vorläufer der Bundesrepublik, dargestellt aus eigenem Miterleben, Frankfurt a. M. 1961, S. 9 f. Ferner W. Köhler, Das Land aus dem Schmelztiegel, Die Entstehungsgeschichte Nordrhein-Westfalens, Düsseldorf 1961, S. $120 \mathrm{f}$. 


\title{
HERMANN PÜNDER
}

POLITIK IN DER

REICHSKANZLEI

\begin{abstract}
Als eine der wenigen „nichtamtlichen" Quellen vermag das mit dem 'Tode Strèsemanns im Oktober 1929 einsetzende Tagebuch des früheren Staatssekretärs in der Reichskanzlei Dr. Pünder zur Geschichte der Großen Koalition und der Ära Brüning eine wesentliche Aussage zu bieten. Dem Leser werden aufschlußreiche Einblicke in den Alltag der obersten Zentralbehörde des Reiches gegeben, und zwar für eine Zeit, da in ihr wirklich noch Politik gemacht wurde. Aus dem unmittelbaren Erleben des Verfassers finden die Verzahnung innen- und außenpolitischer Probleme sowie die wirtschaftlichen Krisenjahre der ausgehenden Republik eine anschauliche Behandlung. Durch Verwendung amtlichen Aktenmaterials hat die Edition eine zusätzliche Abrundung erfahren.

Obgleich Pünder mit dem Sturze Brünings ebenfalls aus dem Reichsdienst ausschied, fallen Streiflichter auch auf die Praxis der Kanzlerschaft Papens, da der Staatssekretär weiterhin in gelegentlicher Verbindung zu seinem Nachfolger Planck blieb. Somit werden die Aufzeichnungen zu einem unentbehrlichen Hilfsmittel für die Beurteilung der letzten Weimarer Jahre.
\end{abstract}


Schriftenreihe der Vierteljahrshefte

für Zeitgeschichte

IN VORBEREITUNG SIND:

LOTHAR GRUCHMANN

Grobraumtheorien des

Dritten Reiches

Die Konstruktion einer

deutschen Monroedoktrin

C. S. LAT OU R

Südtirol und die

Achse Berlin - Rom

\section{Militäropposition} 1938 - 1940

Tagebücher von Oberstleutnant Helmuth Groscurth, herausgegeben von Harold Deutsch und Helmut Krausnick

ENNO GEORG

\section{Die wirtschaftlichen}

Unternehmungen der SS

Jährlich erscheinen zwei

Veröffentlichungen

DEUTSCHE VERLAGS - ANSTALT STUTTGART 